\title{
Libre opinion
}

\section{La défense des revues de sciences humaines et sociales}

\author{
Tracés. Revue de sciences humaines
}

\begin{abstract}
Bien que la revue Natures Sciences Sociétés ne soit pas stricto sensu une revue de sciences humaines et sociales, la rédaction a jugé bon de publier cette pétition, car elle se reconnaît dans bien des arguments qui y sont développés. Ces arguments ont en fait une portée générale et peuvent être appliqués à toutes les revues scientifiques. NSS se sent tout particulièrement concernée. Cette pétition prend d'ailleurs place en France dans tout un mouvement de réflexion et de contestation que les récentes évaluations des revues entreprises par l'AERES ont provoqué. Mais, fait significatif, ce mouvement rejoint les réflexions critiques qui se font jour au niveau international sur l'évaluation scientifique, sur le rôle qu'y jouent les revues et sur la façon de la concevoir et de l'organiser. La publication de cette pétition est une façon d'ouvrir un espace de débat sur ces questions au sein de la revue.
\end{abstract}

La Rédaction

Un débat très important agite, depuis 2008 au moins, la communauté internationale des chercheurs. Il concerne les revues et les modalités de leur classement, de leur notation et de leur évaluation. L'évaluation des revues n'est pas neuve. Le principe en est même acquis pour tous les chercheurs, largement familiarisés avec la logique de hiérarchisation qui sous-tend les pratiques scientifiques. Il est tout à fait normal et sensé de mettre en valeur les revues dont la qualité scientifique est reconnue par les professionnels de la recherche. Mais la signification même des classements français et européen (AERES et ERIH) s'est vue bouleversée par la réforme du statut de l'enseignant-chercheur promue par l'actuel gouvernement, et par le rôle que le ministère de l'Enseignement supérieur et de la Recherche souhaite attribuer à l'AERES (Agence d'évaluation de la recherche et de l'enseignement supérieur).

Pourquoi ces deux visées vont-elles de pair? Précariser le statut de l'enseignant-chercheur et donner à l'AERES le monopole de l'évaluation des chercheurs consiste tout simplement à faire des revues les supports privilégiés de la discrimination et de la compétition entre chercheurs. Une fois la réforme adoptée, ces derniers seront en effet jugés uniquement sur le nombre de leurs publications et sur la note attribuée, par l'AERES, à la revue dans laquelle ils auront publié. Pour le dire autrement, si un chercheur publie un texte dans une excellente revue spécialisée, mais mal (voire pas du tout) classée par l'AERES ou par son aîné l'ERIH (European Reference

Auteur correspondant : Arnaud Fossier, traces@ens-lsh.fr
Index for the Humanities), il ne sera pas considéré comme un «bon» chercheur, et verra son travail confiné aux tâches enseignantes et administratives. Il n'aura donc plus l'occasion de mener à bien ses recherches et de les publiciser.

Cette double réforme est en totale inadéquation avec le métier que nous exerçons. En effet, même si elles reposent sur le principe de la sélection et de la critique constructives, les revues en sciences humaines et sociales n'ont absolument pas vocation à noter les chercheurs! Elles produisent et transmettent un savoir. Qu'elles soient spécialisées, généralistes, interdisciplinaires, leur objectif est d'informer la communauté scientifique, de transmettre de nouveaux programmes de recherche, de poser des problèmes, de donner des idées, de stimuler les interprétations, et non de récompenser ou sanctionner les individus.

La logique comptable et compétitive de l'actuelle réforme met à mal tout particulièrement le rôle des comités de rédaction de ces revues. Ces comités travaillent en effet collectivement à l'élaboration d'une ligne éditoriale, en fonction de laquelle les articles sont sélectionnés ou non pour la publication. Les placer en position de faire le tri entre «bons » et «mauvais » chercheurs, c'est introduire, dans leur travail, d'autres considérations que celles qui président à la ligne éditoriale de la revue. Or les membres d'un comité de rédaction ne sauraient être réduits à la fonction de froids administrateurs, fidèles aux critères de sélection dictés par la mode du moment ou par une conception homogène et stagnante des définitions de la scientificité. 
Une revue n'existe pas non plus sans le travail d'un comité de lecture dont l'avis consultatif ou le pouvoir décisionnel sont absolument cruciaux, comité avec lequel se joue déjà l'évaluation des articles soumis aux revues. Par le profil intellectuel des chercheurs qu'un comité de lecture accueille, comme par les choix argumentés de publication qui sont effectués, le comité de lecture est la clé de voûte d'une revue. Le choix et la définition de ce comité n'est pas une procédure neutre et il n'y a aucune raison pour qu'il en existe une forme unique et supérieure. Là encore, se joue l'identité d'une revue. La course à la publication, le risque de la compilation hétéroclite, l'accumulation de critères de sélection mal ajustés aux situations spécifiques : voilà ce que propose aujourd'hui le ministère de la Recherche aux revues dont certaines sont pourtant mondialement réputées pour leurs qualités scientifiques et l'originalité de leur ligne éditoriale.

Voulons-nous de cette terrifiante homogénéisation du champ des revues? Voulons-nous que les revues deviennent les «chambres d'enregistrement » des ambitions individuelles des chercheurs? Non, car cette logique compétitive et quantitative correspond mal aux temporalités des chercheurs en sciences humaines et sociales. Faire du terrain, aller aux archives, formuler de nouvelles hypothèses, proposer des interprétations, écrire, et penser, tout cela prend du temps ! À l'inverse, être condamné à publier à tout prix, n'importe où, n'importe quand afin d'éviter la relégation dans la catégorie "mauvais chercheur » est tout simplement incompatible avec les exigences d'un travail de recherche honnête.

Enfin, les mutations actuelles de l'Université font peser un grand nombre d'incertitudes sur l'avenir financier et matériel de la plupart des revues. Beaucoup d'entre elles étant liées à des institutions, des laboratoires, des centres de recherche, amenés à être restructurés si l'ANR (Agence nationale de la recherche) en décide ainsi, elles risquent clairement leur survie! En France, si un laboratoire meurt, une revue meurt. Si le CNRS meurt, des dizaines de revues meurent. Ce n'est donc pas "un peu» de la recherche "qui s'en va», mais bien une part massive des supports de la recherche qui disparaît!

Dans le contexte d'un tarissement évident des abonnements de bibliothèques et d'une baisse non moins évidente des ventes de sciences humaines et sociales en librairie, les revues se retrouvent clairement confrontées au problème (que subit également la presse écrite) du modèle, devenu économiquement le plus viable, de la numérisation. En dépit de l'existence d'excellents portails électroniques comme Cairn, Revues.org ou Persée, la France, faute de prise de conscience politique sur le sujet, accuse un retard considérable par rapport aux pays étrangers.

On nous dit que la revue va devenir le moyen central de l'évaluation des chercheurs, mais on ne songe même pas à ce qu'est réellement une revue de sciences humaines. On en ignore farouchement les modes de fonctionnement, les usages, l'originalité éditoriale, les soutiens et les modes de financement. Mais ceci, finalement, n'étonnera guère, puisque force est de constater que le gouvernement actuel veut engager à toute vitesse la réforme de la recherche, sans même avoir pris le temps d'en connaître ni les acteurs ni les supports. Nous, revues de sciences humaines et sociales, appelons donc à une suppression du classement AERES, dans le prolongement de la demande de moratoire du 9 février 2009 par les instances scientifiques du CNRS. Nous demandons la discussion collective, par les chercheurs, de nouveaux critères d'évaluation des revues, leur transparence totale, et le maintien de leur pluralité (afin que les spécificités des revues scientifiques soient reconnues en tant que telles). Enfin, nous exigeons que les revues ne soient pas les nouveaux vecteurs de la notation des chercheurs ${ }^{1}$.

Pour signer : http://www.shesp.lautre.net/spip.php? article 46

\footnotetext{
${ }^{1}$ Ce texte est la reproduction exacte de la pétition publiée sur Internet le 18 février 2009. Le site indiqué à la fin en donne la version mise à jour le 31 mars 2009.
} 